\title{
Inflection of In-Situ Ergonomics in Service Engineering
}

\author{
Atsushi Shinya \\ Systems Business Unit. Shimadzu Business Systems Corporation, \\ 1, Nishinokyo-kuwabaracho, Nakagyo-ku, Kyoto 604-8442 Japan \\ shinyashimadzu.co.jp
}

\begin{abstract}
In-Situ Ergonomics" which extended the direct observation method was discussed. It is the method of using a sensor for data collection. By observing all the situations in which a user works for a long period of time, this method can be used in order to improve service. As an example, the use situation of the electric pot with a communication function was analyzed. Various users' use situation over one electric pot was clarified. And the improvement proposal of service was shown.
\end{abstract}

Keywords: In-Situ Ergonomics, direct observation method, sensors, datamining.

\section{Introduction}

Process P and Process D in a PDCA (Plan - Do - Check - Action) cycle have so far been well examined by service engineering. From now, the study of Process $\mathrm{C}$ and Process A will become more important. The Process $\mathrm{C}$ and Process A are input data of the next cycle of PDCA cycle(Fig. 1).

Quality of output cannot exceed quality of input. This paper describes research of the improvement method of Process $\mathrm{C}$ and process $\mathrm{A}$.

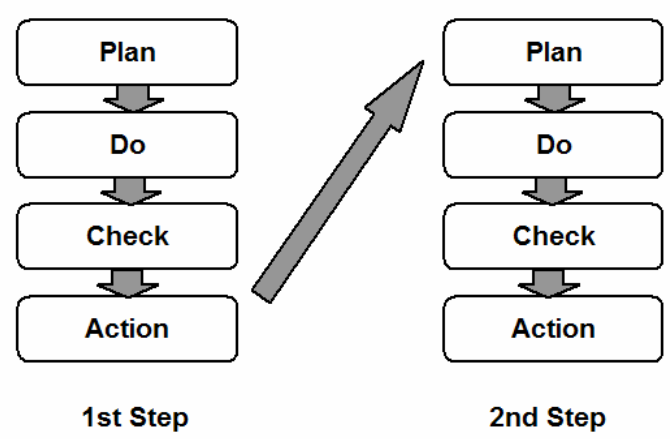

Fig. 1. PDCA Cycle 


\section{Direct Observation Method}

The direct observation method is an objective method of collecting data.

The direct observation method involves the investigator witnessing the user in the environment where the product is used and recording the use over several hours or several days. This method has the benefit of being cheap, as it does not require especially high plant and equipment costs.

Questionnaires are a well-known method of surveying users. As a questionnaire poses questions set by the investigator, it can only obtain replies within the scope hypothesized by the investigator. Questionnaires are a method of extracting problems from the viewpoint of the investigator.

On the other hand, the direct observation method observes the user's actions to discover any problem points. It can therefore obtain results more closely matching the user, such as discovering unexpected ways of operation the user may adopt.

The direct observation method allows the investigator to discover and compile information on a user's subconscious behaviors. Consequently, it allows discovery of service problems that the user does not sense as a problem.

The direct observation method can obtain more profound user information but it is not without its shortfalls.

The first is the high personnel costs. The investigator stays in the subject's house and captures information on the user's behavior on a one-to-one basis. This contrasts with questionnaires that work on a one-to-many basis and simply require the replies to be recovered. The amount of data compiled in unit time is significantly higher using a questionnaire.

As the user controls the operations with the direct observation method, there is no guarantee that the user will use the service anticipated by the investigator during the period of the investigation. Even if the service is used, it cannot be guaranteed that this covers the entire usage pattern for that service by the user.

Also, with observations of just a single user, it is impossible to generalize the problem points with the service. As number of samples is small, the proportion of all users having that problem is unknown. Consequently, it is not possible to sort multiple problems in order of frequency of occurrence at the planning stage to determine countermeasures.

If the investigator discovers an unexpected problem through observations of a single user, there is a good possibility that the service has other problems.

The author has proposed In-Situ Ergonomics to compensate for the shortfalls of the direct observation method.

\section{In-Situ Ergonomics}

The ergonomic study domain apply in a PDCA cycle of service. In-Vitro Ergonomics will be applied in Process $\mathrm{P}$ and Process D. And In-Situ Ergonomics will be applied in Process $\mathrm{C}$ and Process $\mathrm{A}$. 


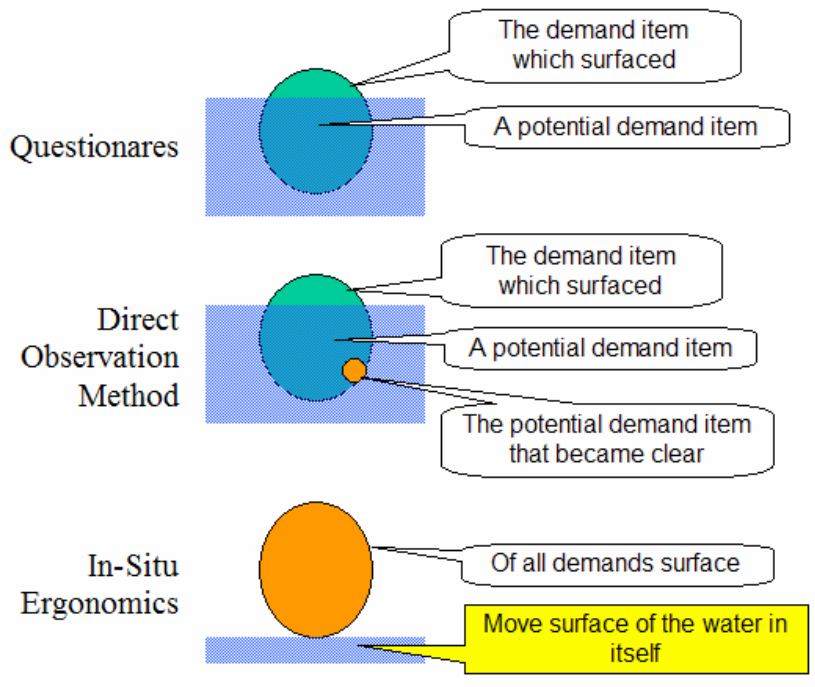

Fig. 2. The Iceberg Problem

Until now, the ergonomic study has been performed between Process $\mathrm{P}$ and Process D. In the beta test phase like Process $\mathrm{P}$ and Process D, a researcher researches for improvement of services.

Technology evolves now.

Various sensors can collect a product and information of the use spot of service. And information can transmit a message in the company side via a network. It is used for remote monitor of a control unit. And it is used as monitor information of a senior person, too.

For example,

- opening and shutting information of a door with microswitch

- On/Off of illumination using a pyro-electric sensor

- consumption information of water of a restroom with an automatic faucet.

The many information is provided. An ergonomic method called as "In-Situ Ergonomics", it collects and analyses data from a provided sensor in a product and service on the site. In other words, it becomes direct observation method with a sensor.

In-Situ Ergonomics solves "The Iceberg Problem" (Fig. 2).

By the direct observation method, a researcher and a user investigate it by 1 to 1 .

By In-Situ Ergonomics method, it is advantageous in that following

- It can collect mass information at a time. It collects the information with a sensor containing it in product oneself via a network. This method can collect information of all the products in the using spot. 
- In-Situ Ergonomics can conduct a continuous investigation. Whenever a user uses a product, information is dispatched automatically by a product installed in a product use place. On this account this method can investigate an action of a user continuously.

By these advantages,

- The researcher does not participate in data collection

- Large human reduction in cost is possible

- This method does not perform artificial data collection, it can collect an objective fact than conventional ergonomic study methods.

However, there are the following weak points in this method.

- Initial cost is big. Infrastructure maintenance such as in-home networks is necessary.

In-Situ Ergonomics applies service, and it is the method that is necessary to be improved.

\section{The Service Engineering Application Example That Using In-Situ Ergonomics Method}

The electric pot which has communication function were used. The use situation of 3,000 electric pots was investigated. A period is one month. Based on provided information, "The service that is serving hot water " is researched.

This service is called "i-Pot" and it is provided by ZOJIRUSHI. It is the service which watches elderly people from a remote place.

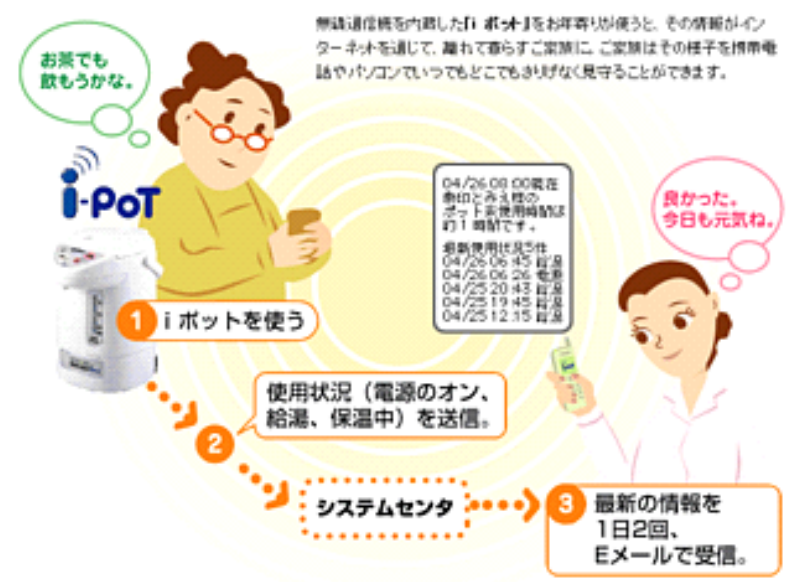

Fig. 3. The "i-Pot" service. (www.mimamori.net). 
Each event of the "power supply ON (ps)" of an electric pot, "hot-water supply (hw)", and a "keeping-warm state (kw)" is acquired, and it transmits to a server through a communication line in real time.

A "keeping-warm state" is an event automatically transmitted for every hour, when an electric pot is in a turning-on-electricity state. A server sends the event information on an electric pot by E-mail according to a demand of the family of a remote place. Thereby, it enables the family of a remote place to watch elderly people's condition nonchalantly.

With this service, a user acquires the event of the electric pot currently used usually. For this reason, it is an advantage that a mental burden is not placed on the user side. However, the user using an electric pot is not necessarily elderly people. Moreover, it cannot be concluded that one person uses.

In other words, all the events of all the users using the electric pot can be acquired.

\subsection{Data Specification}

Although an electric pot transmits event information to a server in real time, the information summarized per 10 minutes here is used. This is equivalent to performing the time specimen method in a direct observation method in observation unit 10 minutes. The priorities of an event are 1. hot-water supply, 2. power supply ON, and 3. keeping warm.

It is represented with hot-water supply event when hot-water supply event and the power supply $\mathrm{ON}$ event occur in observation unit time.

The communication line is using "DoPa" of NTT DoCoMo.

\subsection{Survey Content}

It was observed by the In-Situ Ergonomics method for one month from November 1, 2006 to November 30. The number of object electricity pots was about 3,000 sets.

However, since there were an addition of a new contract person, a unused day, etc., the number of operation of day by day was changed.

Moreover, a user's attribute is unknown entirely. It is not used this time.

The calendar of Japan in November, 2006 is shown below. * The mark is a public holiday.

Table 1. 2006/11 of Calendar (Japan)

\begin{tabular}{|c|c|c|c|c|c|c|}
\hline Sun & Mon & Tue & Wed & Thu & Fri & Sat \\
\hline & & & 1 & 2 & $* 3$ & 4 \\
\hline 5 & 6 & 7 & 8 & 9 & 10 & 11 \\
\hline 12 & 13 & 14 & 15 & 16 & 17 & 18 \\
\hline 19 & 20 & 21 & 22 & $* 23$ & 24 & 25 \\
\hline 26 & 27 & 28 & 29 & 30 & & \\
\hline
\end{tabular}

\subsection{Overall Result}

The acquired data was registered and analyzed to "Microsoft SQL Server 2000" which is a database management system. From the obtained result, fundamental statistical information is shown below. 


\subsubsection{All the Acquisition Data Numbers}

\section{$12,635,568$ events}

\subsubsection{The Number of Electric Pots (= the Number of Users)}

3,172 units

\subsubsection{Use Days Distribution Classified by Electric Pot}

Moreover, it is as follows when it displays in calendar form.

Table 2. Use days - number of electric pots

\begin{tabular}{|l|l|l|l|l|l|}
\hline Use days & \#pots & Use days & \#pots & Use days & \#pots \\
\hline 30 & 2492 & 20 & 13 & 10 & 6 \\
\hline 29 & 153 & 19 & 9 & 9 & 11 \\
\hline 28 & 79 & 18 & 17 & 8 & 2 \\
\hline 27 & 42 & 17 & 11 & 7 & 3 \\
\hline 26 & 35 & 16 & 4 & 6 & 9 \\
\hline 25 & 30 & 15 & 9 & 5 & 11 \\
\hline 24 & 25 & 14 & 8 & 4 & 8 \\
\hline 23 & 12 & 13 & 4 & 3 & 8 \\
\hline 22 & 15 & 12 & 6 & 2 & 8 \\
\hline 21 & 19 & 11 & 8 & 1 & 115 \\
\hline
\end{tabular}

Note: Use days $30=$ he/she used a pot everyday for 1 month

Table 3. Number of electric pots(Calendar form)

\begin{tabular}{|l|l|l|l|l|l|l|c|}
\hline Sun & Mon & Tue & \multicolumn{1}{|c|}{ Wed } & \multicolumn{1}{|c|}{ Thu } & Fri & Sat & \\
\hline & & & 1 & 2 & $* 3$ & 4 & Day \\
\hline & & & 2905 & 2905 & 2904 & $\underline{2885}$ & \#ope \\
\hline 5 & 6 & 7 & 8 & 9 & 10 & 11 & Day \\
\hline 2894 & 2909 & 2897 & 2909 & 2916 & 2911 & 2913 & \#ope \\
\hline 12 & 13 & 14 & 15 & 16 & 17 & 18 & Day \\
\hline 2914 & $\underline{3016}$ & 2937 & 2922 & 2937 & 2929 & 2929 & \#ope \\
\hline 19 & 20 & 21 & 22 & $* 23$ & 24 & 25 & Day \\
\hline 2920 & 2930 & 2929 & 2926 & 2931 & 2932 & 2932 & \#ope \\
\hline 26 & 27 & 28 & 29 & 30 & & & Day \\
\hline 2940 & 2941 & 2947 & 2943 & 2944 & & & \#ope \\
\hline
\end{tabular}

Note:

The number of one-day average operation 2,924 sets

The number of the one-day maximum operation 3,016 sets

The number of the one-day minimum operation $\quad 2,885$ sets 
Here, the concrete electric pot use day in 115 electric pots which use only one day in November was as follows.

Table 4. Day - Number of electric pots

\begin{tabular}{|c|r|r|r|r|r|r|}
\hline Day & \multicolumn{1}{|c|}{$11 / 01$} & $11 / 13$ & $11 / 14$ & \multicolumn{1}{c|}{$11 / 16$} & $11 / 20$ & $11 / 30$ \\
\hline \#ope & 2 & 100 & 8 & 1 & 1 & 3 \\
\hline
\end{tabular}

\subsection{The Result According to Electric Pot}

The statistics result according to electric pot is shown below.

\subsubsection{Number of Times of Hot-Water Supply Event by Power Supply Event}

The following is the whole number-of-times statistics value of hot-water supply for every power supply injection.

Power supply injection (ps)

Hot-water supply (hw)

hw / ps
75,615 ("hot-water supply" is overwrited) 444,169

5.87

\subsubsection{The Power Supply Injection Hot-Water Supply Ratio Classified by}

\section{Electric Pot}

A power supply ON (ps) hot-water supply (hw) ratio is taken out for every electric pot.

The statistical information is shown.

Table 5. hw - ps ratio

\begin{tabular}{|l|l|l|l|}
\hline hw / ps & \# of pot & hw / ps & \# of pot \\
\hline$<1.0$ & $\underline{98}$ & $10.0-20.0$ & 213 \\
\hline $1.0-2.0$ & 224 & $20.0-30.0$ & 109 \\
\hline $2.0-3.0$ & 207 & $30.0-40.0$ & 85 \\
\cline { 2 - 4 } $3.0-4.0$ & 224 & $40.0-50.0$ & 73 \\
\cline { 2 - 4 } $4.0-5.0$ & 219 & $50.0-60.0$ & 65 \\
\hline $5.0-6.0$ & 196 & $70.0-80.0$ & 38 \\
\hline $6.0-7.0$ & 151 & $80.0-90.0$ & 28 \\
\hline $7.0-8.0$ & 102 & $90.0-100.0$ & 32 \\
\hline $8.0-9.0$ & 84 & $>100.0$ & 292 \\
\hline $9.0-10.0$ & 71 & & \multicolumn{2}{|l}{} \\
\hline
\end{tabular}

One example of less than 1.0 electric pot is shown. It is a pattern on November 1 of a certain electric pot. 
Table 6. A pot event of 2006/11/01

\begin{tabular}{|l|l|l|l|l|l|}
\hline Time & event & Time & event & Time & event \\
\hline $1: 30$ & ps & $6: 40$ & hw & $16: 30$ & ps \\
\hline $1: 50$ & hw & $9: 00$ & ps & $17: 00$ & hw \\
\hline $4: 30$ & ps & $12: 50$ & hw & $17: 50$ & ps \\
\hline $4: 40$ & hw & $13: 00$ & hw & $18: 10$ & hw \\
$6: 20$ & ps & $13: 40$ & ps & & \\
\hline
\end{tabular}

\subsection{Consideration}

A characteristic day can be extracted by seeing the number of operation according to day. Though there is a day which uses an electric pot or is not used by people, if it sees on the whole, it will almost be changeless in the number of operation.

It is over 3,016 sets and 3,000 sets only 11/13. When this cause was explored, there is an electric pot currently used only one day during November, and it became clear that 100 sets of them were 11/13. It is thought that it is based on a sudden factor of some kind.

Next, hot-water supply is observed. All the users' number of times of average hotwater supply was 17,624 times, most number of times of hot-water supply was 18,393 times $(11 / 19$, Sun), and the smallest number of times of hot-water supply was 16,727 times $(11 / 1$, Wed). It seems that a holiday has generally much number of times of hotwater supply.

The combination of a power supply injection and hot-water supply is considered. By this data, when hot-water supply and a power supply injection are in an observation unit, hot-water supply is adopted.

If it is directions of the usual electric pot, water will be supplied first. Next, a power supply is switched on. And, hot-water supply should continue until hot water is lost.

In this case, it is thought that several times per power supply injection of hot-water supply occurs. So, when the number of times of hot-water supply per number of times of a power supply injection is less than 1.0, it is thought that it is a certain special use pattern.

The example of one day of a certain electric pot is shown as the example of representation (Table 6). From this pattern, there is no concept of keeping warm and it is thought that the power is always turned off after use. In this user, it is thought that it is used not as an electric pot but as an electric kettle. It is recommending the electric kettle in which the proposal of the optimal hot-water supply service has a function a power supply being automatically disconnected after boil, to this user.

Thus, if Plan of the following cycle in PDCA is performed taking advantage of these knowledge, it is possible to draft a high-precision plan.

\section{Conclusion and Proposal}

This paper described the concept of the "In-Situ Ergonomics" method that uses sensors to extend the direct observation method. It was shown that In-Situ 
Ergonomics can solve the problems of cost and investigation period associated with the conventional direct observation method.

In-Situ Ergonomics aims to improve the accuracy of the inputs in ergonomics research. Some results from conventional ergonomics research were not necessarily practical due to the inaccuracy of the information at the input stage.

With conventional ergonomics methods, no particular problems are associated with the analysis and outputs after the inputs are complete. Therefore, applying the In-Situ Ergonomics method should enhance the overall level of ergonomics research.

Acknowledgements. I am thankful to Mr. Shinya of ZOJIRUSHI.

\section{References}

1. Nakazawa, et al.: Shinrigaku Manual the Observation Method, Kitaohji-Syobo (1997)

2. Shinya, et al.: In-situ ergonomics: proposal for in-situ ergonomics evaluation method, IEA2003 (Korea) (2003)

3. Shinya, et al.: In-Situ Ergonomics: A proposal of product operation information gathering method for ubiquitous computing environmen, HFES2004 (2004)

4. Shinya, Yamaoka.: Datamining of Universal Access Log by using In-Situ Ergonomics Method, HCI International 2005 (2005) 